\title{
On the Performance of RLNC based Wireless Broadcasting with Uniformly Distributed Users
}

\author{
Xiaoli Sun ${ }^{1, a^{*}}$, Youyun $\mathrm{Xu}^{2, \mathrm{~b}}$, Xiaojun Liu ${ }^{3, \mathrm{c}}$, Wenfeng Ma, ${ }^{4, \mathrm{~d}}$, and Kui $\mathrm{Xu}^{5, \mathrm{e}}$ \\ 1,2,3,4,5 Institute of Communications Engineering, PLA University of Science and Technology, \\ Nanjing, China \\ agdxsunxiaoli@sina.com, ${ }^{\mathrm{a}}$ Igdxxukui@126.com,
}

\begin{abstract}
Keywords: Wireless broadcasting system, random linear network coding, erasure channel, performance analysis.

Abstract. In this paper, we analyze the performances of the random linear network coding (RLNC) based wireless broadcasting system, where a source wants to broadcast $N$ packets to a large number of users with uniform distribution. By resorting to stochastic geometry analysis, we deduce the approximate average number of transmissions and the average percent of successful decoding users in the RLNC based wireless broadcasting system. Simulation results are given to demonstrate the accuracy of our theoretical analyses and the performance improvement of using RLNC scheme when compared with LT codes. Meanwhile, we show that a user can recover all the original packets with high probability by receiving $N$ out of all the transmitted RLNC combined packets.
\end{abstract}

\section{Introduction}

Broadcast is an important mechanism in wireless networks to transmit common data to multiple users. One major challenge for broadcast traffic in wireless networks is that wireless links are usually unreliable, and their qualities differ from user to user.

The common solution to guarantee reliable deliveries over unreliable wireless links is the Automatic Repeat reQuest(ARQ). With ARQ, receivers afford feedback to the source, using either acknowledgements (ACKs), if the packets are correctly received, or negative acknowledgements (NACKs), if the packets are deemed to be erroneous. However, as the overhead of gathering feedback information increases with the size of the network, using ARQ for wireless broadcasting can incur significant delay and is not scalable. Thus, a solution for wireless broadcasting that does not require feedback information is needed.

Recent works have shown that the transmission efficiency and reliability of wireless broadcast can be enhanced by employing network coding. Network coding, which was proposed by Ahlswede, has been demonstrated as a promising approach to improve throughput in wireless networks. It has been proved that for many situations such as multicast and broadcast, using appropriate encoding schemes can achieve the network capacity [1].

\section{A. Motivation and Related work}

Rateless codes, which have been proven effective for wireless broadcasting systems that do not require explicit receiver feedback, can automatically adapt to any channel conditions and avoid the need for feedback channels [2]. Rateless codes can generate a potentially limitless stream of coded packets. The Luby transform (LT) codes [3], which adopted the idea of random encoding, is one of the rateless codes.

Recently, RLNC has gained popularity as an useful performance-enhancing tool for wireless networks $[4,5,6]$, wherein all the coefficients are selected at random from a large Galois field, can give innovative information with high probability.

Authors in [7] propose a wireless broadcast retransmission scheme based on random linear network coding, wherein the source combines all lost packets to a single one by linear network coding for retransmission according to the updated feedback matrix $\mathbf{T}$. Then receivers are able to decode the lost packets by method of Gaussian Elimination when they receive enough coded packets. But this strategy is still based on ARQ. In many literatures, the erasure probabilities of different users are assumed to be 
the same for convenience[8], which is not in conformity with the reality. In [9], the probability under a certain outage fraction (number of users that do not decode the $N$ original packets) is derived. In the practical wireless broadcasting system, the delay and transmission efficiency performances are more important. So we deduce the approximate average number of transmissions and the average percent of successful decoding users in this paper.

\section{B. Contribution}

In this paper, we make a performance analysis on a wireless broadcasting system, wherein the erasure channel qualities are differ from user to user and the overhead caused by feedback signals can be reduced since we do not need feedback from receivers to the source. For the wireless broadcasting system with uniformly distributed users, the probability that a user can successfully receive a packet during any time interval is decrease with the increasing of the distance $r$. The following is a detailed summary of our contributions:

1) The performance of RLNC based wireless broadcasting system, where one source broadcasts packets to a large number of users with uniform distribution, is analyzed. The average percent of successful decoding users (when the transmitted encoded packets are fixed) and the average number of transmissions are obtained analytically.

2) Based on the above analysis, we derive an analytic approximation to the average number of transmissions and the average percent of successful decoding users by resorting to stochastic geometry theory.

3) Simulations are conducted to validate both the accuracy of the theoretical analysis and the performance improvement of using RLNC scheme compared with LT codes.

The rest of this paper is organized as follows. In Section II, we provide a system model for network coding in a broadcast scenario over erasure channel whose qualities differ from user to user with no feedback channel. In Section III, we derive an analytic approximation to the average percent of successful decoding users and the average number of transmissions. The accuracy of the analysis using computer simulation is shown in Section IV, and followed by the conclusions in Section V.

\section{Broadcasting with Network Coding}

\section{A. System Model}

We consider a wireless broadcast system consisting of one source and $K$ receivers/users. We denote $S$ as the source node and $U^{i}$ as the $i$-th user, where $i \in\{1,2, \ldots, K\}$. The source wants to send a file which is divided into $N$ information packets, to all users via a wireless channel, which is modeled as an independent packet erasure channel. Without loss of generality, we assume that the information packets have equal length. Each packet transmitted by $\boldsymbol{S}$ is obtained by linearly combining the $N$ original packets, with coefficients drawn from $\mathrm{GF}(q)$.

The users are assumed to be uniformly distributed with a density of $\rho$ (number of users per square meter) throughout $S$ 's coverage area, over a radius $R$, as is shown in Fig. 1. Assuming $K$ is large enough, the user density and the number of users have such a relationship: $\rho=\frac{K}{\pi R^{2}} . S$ seeks to broadcast the $N$ information packets to the users via suitable encoding, without any feedback from users. We try to find the average percent of successful decoding users and the average number of transmissions under the RLNC based broadcast scheme. For transmission power $P$, bit rate $R_{0}$, system bandwidth $W$ (in $\mathrm{Hz}$ ), noise power spectral density $\frac{N_{0}}{2}$, block fading factor $\mu$, and $\alpha$ as the path loss exponent, the erasure probabilities of any individual packet over an erasure channel from $S$ to a user (at a distance of $r$ to $S$ ) during any time interval is given by [10]:

$$
p(\mathrm{r})=1-e^{-\frac{\left(2 \frac{2 R_{0}}{W}-1\right) N_{0} W}{\mu r^{-\alpha} P}}
$$


For each transmission, a node may or may not receive the transmitted packet successfully, as modeled in Eq. (1). If a packet is erased, it will be discarded. Otherwise, the packet is assumed to be successfully received.

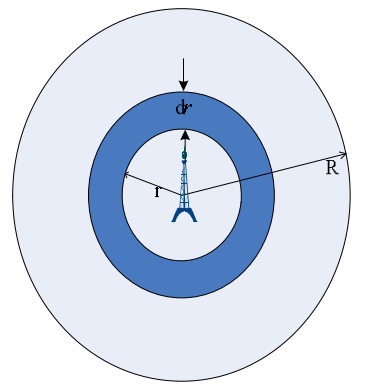

(a)

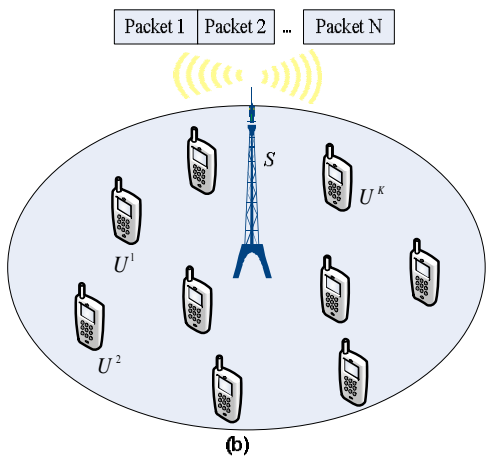

Fig. 1. A source serving all the users in its coverage range of $R$ with user density $\rho$. Users are locating in rings centered at $S$ with infinitesimal width.

\section{B. Random Linear Network Coding}

In traditional fountain codes, source packets are combined over a binary field (or Galois field $F_{2}$ ) [11]. In the RLNC scheme, a set of $N$ packets $\left\{x_{1}, x_{2}, \mathrm{~K}, x_{N}\right\}$ with the same length needs to be transmitted. $S$ encodes all the packets by random linear coding, generating a new coded packet: $y_{i}=\sum_{j=1}^{N} g_{i j} x_{j}$, the coding coefficients $g_{i j}(1 \leq j \leq N)$ are chosen randomly from a finite field of size $F_{q}$. In other words, each transmitted packet is a linear combination of the $N$ uncoded packets, and the $N$ coefficients are represented by a $1 \times N$ vector which is called the encoding vector of that packet. When a coded packet is broadcast, its encoding vector will be attached in the header and broadcast at the same time. Therefore, a coded packet is composed of $l=h+n+N g$ bits, as is shown in Fig. 2, where $h$ represents the number of bits allocated for the packet's header, $n$ is the number of bits for the linear combination of all $N$ original packets, and $g=\log _{2} q$ is the number of bits used to represent the randomly chosen coding coefficients for each original packet. Obviously, RLNC systems incur additional header. However, if the size of the information packets is reasonably large, the additional overhead is acceptable.

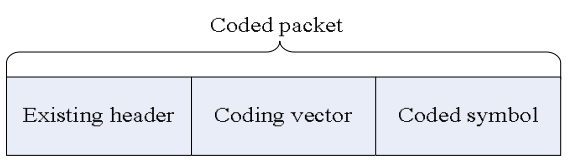

Fig. 2. Structure of the encoded packet

For $U^{i}$, we use $M^{i}$ to denote the number of encoding vectors it has received. By putting these $M^{i}$ vectors together, we obtain an $M^{i} \times N$ matrix $C^{i}$, which is called encoding matrix of $U^{i}$. As the relationship among $\boldsymbol{C}, \boldsymbol{Y}$, and $\boldsymbol{X}$ can be expressed as: $\boldsymbol{Y}=\boldsymbol{C} \cdot \boldsymbol{X}, \boldsymbol{X}=\left[x_{1}, x_{2}, \mathrm{~L}, x_{N}\right]^{T}, \boldsymbol{Y}=\left[y_{1}, y_{2}, \mathrm{~L}, y_{M}\right]^{T}$, In random linear network coding, a variant of the Gaussian Elimination called progressive decoding is widely used to calculate, i.e.,

$$
\left[\begin{array}{l}
x_{1} \\
x_{2} \\
\mathrm{M} \\
x_{N}
\end{array}\right]=\left(\begin{array}{rrr}
g_{11} & \mathrm{~L} & g_{1 N} \\
\mathrm{M} & \mathrm{O} & \mathrm{M} \\
g_{M 1} & \mathrm{~L} & g_{M N}
\end{array}\right)^{-1}\left[\begin{array}{l}
y_{1} \\
y_{2} \\
\mathrm{M} \\
y_{M}
\end{array}\right]
$$

If the rank of $U^{i}$ s encoding matrix $C^{i}$ is $N$, it can decode the original packets, and we say that $U^{i}$ is complete. Otherwise, we say that $U^{i}$ is incomplete. 
Using random linear coding arguments [12], the probability that the original packets can be decoded from any $N$ RLNC encoded packets (the rank of $\boldsymbol{C}^{i}$ is $N$ ) is equal to $\left(1-\frac{1}{q^{N}}\right)\left(1-\frac{1}{q^{N-1}}\right)\left\llcorner\left(1-\frac{1}{q}\right)\right.$, which is bounded from below by $\left(1-\frac{1}{q-1}\right)$. Therefore, for a large enough field size $q$ (e.g., $q \geq 8$ ), it is sufficient for the users to receive approximately $N$ coded packets to be able to decode the original packets.

\section{The Statistical Analysis of RLNC Broadcasting}

Based on the above discussion, we assume that, under the RLNC scheme, the source continues to transmit encoded packets until each user successfully receives $N$ linear combinations. $S$ transmits $t \geq N$ encoded packets; a receiver needs to successfully receive at least $N$ packets in order to be able to recover (with high probability) the original information. Therefore, the probability that a user located at a distance $r$ from $S$ is able to decode the original message, can be calculated as follows:

$$
P(r)=\sum_{\tau=N}^{t}\left(\begin{array}{l}
\tau-1 \\
N-1
\end{array}\right)(p(r))^{\tau-N}(1-p(r))^{N}
$$

Let $C I$ be the random variable representing the total number of required transmissions under the condition that all users are promised to decode the original packets, $f$ representing the number of users who can decode the full information when the transmitted encoded packets $t$ are fixed. In this section, we derive the relation between the total number of required transmissions $C I$ and the number of information packets $N$, the relation between the average percent of successful decoding users $\eta$ and the number of transmitted packets $t$. We divide a finite coverage area of radius $R$ into $Q$ equal concentric annuli and the width of each annulus equals $\frac{R}{Q}$, as is shown in Fig. 1(a). The inner and outer radius of the $i$-th annulus is thus $\frac{(i-1) R}{Q}$ and $\frac{i R}{Q}$, respectively. We assume $Q$ is large enough so that the encoded packets that transmitted to the users in the $i$-th annulus experience almost the same erasure channel and hence the decoding probability for any user within an annulus can be treated as identical $P\left(r_{i}\right)=P\left(\frac{i R}{Q}\right)$. Let $K_{i}$ be the number of users in the $i$-th ring, then $K_{i}=2 \pi r_{i} \rho d r$.

Let $f\left(r_{i}\right)$ be a random variable denoting the number of users in the $i$-th ring who can decode the full information. $f\left(r_{i}\right)$ follows binomial distribution, i.e.,

$$
P_{U}\left(f\left(r_{i}\right)=m\right)=\left(\begin{array}{l}
K_{i} \\
m
\end{array}\right) P\left(r_{i}\right)^{m}\left(1-P\left(r_{i}\right)\right)^{K_{i}-m}
$$

As we all known a Binomial variable with probability of success $p$ and number of trials $x$ can be approximated as a Poisson variable with parameter $\lambda=x p$ when $x$ is large enough. Therefore, treating $f\left(r_{i}\right)$ as a Poisson variable with parameter $\lambda_{i}=K_{i} P\left(r_{i}\right)$, the total number of the users that can recover the information packets is: $f=\sum_{i=1}^{o} f\left(r_{i}\right) \cdot f\left(r_{i}\right)$ is independent Poisson random variable with parameter $\lambda_{i}$, therefore $f$ is a Poisson variable with parameter $\lambda=\sum_{i=1}^{o} \lambda_{i}$. The average number of users who can decode the information packets after $t$ transmission is $\mathrm{E}[f]=\lambda$. When $Q$ approaches infinity, we have:

$$
\lambda=\lim _{Q \rightarrow+\infty} \int_{0}^{R} 2 \pi r \rho P(r) d r
$$

So that the average percent of successful decoding users, i.e., 


$$
\eta=\frac{\lambda}{K}
$$

In respect that all users are promised to decode the original packets, the total number of required transmissions should be given as follows:

$$
\begin{aligned}
& C I=N+\sum_{t=N}^{\infty}\left(1-\prod_{j=1}^{K} P^{j}(r)\right)=N+ \\
& \sum_{t=N}^{\infty}\left\{1-\prod_{j=1}^{K}\left[\sum_{\tau=N}^{t}\left(\begin{array}{c}
\tau-1 \\
N-1
\end{array}\right)\left(p^{j}(r)\right)^{\tau-N}\left(1-p^{j}(r)\right)^{N}\right]\right\}
\end{aligned}
$$

where $P^{j}(r)$ represent the probability that user $j$ located at a distance $r$ from $S$ is able to decode the original message.

Let

$$
w=\prod_{j=1}^{K}\left[\sum_{\tau=N}^{t}\left(\begin{array}{l}
\tau-1 \\
N-1
\end{array}\right)\left(p^{j}(\mathrm{r})\right)^{\tau-N}\left(1-p^{j}(r)\right)^{N}\right]
$$

Then,

$$
\log _{2}(w)=\sum_{j=1}^{K} \log _{2}\left(P^{j}(r)\right)
$$

when $Q$ and $K$ are large enough and the users are uniformly distributed with a density of $\rho$, the decoding probability for any user within an annulus can be treated as identical $\not\left(r_{i}\right)=P\left(\frac{i R}{Q}\right)$. According to Eq.(9):

$$
\log _{2}(w)=\int_{0}^{R} \rho 2 \pi r \log _{2}(P(r)) d r
$$

Hence, the total number of required transmissions can becalculated as

$$
C I=N+\sum_{t=N}^{\infty}\left(1-2^{\int_{0}^{R} \rho 2 \pi r \log _{2}(P(r)) d r}\right)
$$

Hence the average number of required transmissions in $S$ - $U$ is, i.e.,

$$
\overline{C I}=\frac{C I}{N}
$$

Table 1. The Simulation Parameters

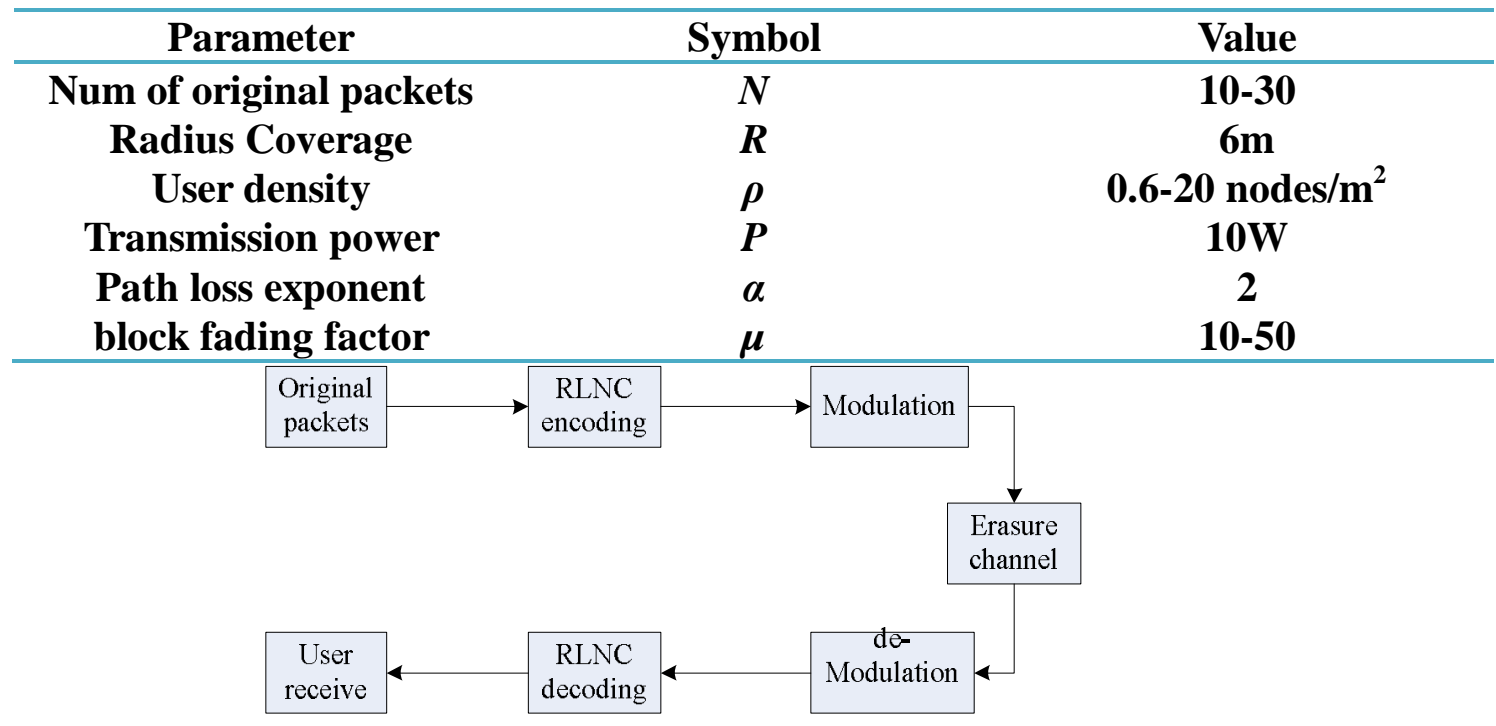

Fig. 3. System block diagram used in the simulation. 


\section{Evaluation Results}

In this section, we evaluate the accuracy of our approximation in Eq. (6) (12) via computer simulation. Then, we compare the performance of RLNC with LT codes based broadcast over erasure channels. The simulation parameters and system block diagram are presented in Table1 and Fig. 3 respectively. $S$ broadcasts coded packets using binary phase shift keying (BPSK) modulation; We model the channel between $S$ and the users in its coverage area as independent Rayleigh blockfading channel where each block is composed of a packet with the length of $l$ bits. As is depicted in Fig. 3, if the transmission bit rate is larger than the instantaneous channel capacity, the packet is erased.

A. Accuracy of the Approximation

For a fixed number of transmissions packets $t$, the average percent of successful decoding users $\eta$ is presented in Fig. 4. As can be seen, our analysis in Eq. (6) follows the simulation results closely.

The erasure probability $p(r)$ in Eq. (1) depends on other parameters such as path loss exponent $\alpha$ and block fading parameter $\mu$. Fig. 4 presents the effect of $\mu$ on $\eta$ and the approximation accuracy in Eq. (6). For a fixed $r$, as $\mu$ increases, the erasure probability $p(r)$ decreases. In other words, increasing $\mu$ improves the reliability of the erasure channel between $\mathrm{S}$ and the users. Hence, for a fixed $t$, as $\mu$ increases, $\eta$ increases.

For a fixed number of original packets $N$, Fig. 5 and Fig. 6 present the average number of required transmissions $\overline{C I}$. As can be seen, our analysis in Eq. (12) follows the simulation results closely.

Fig. 5 presents the effect of the block fading parameter $\mu$ on $\overline{C I}$ and the approximation accuracy in Eq.(12). According to what we discussed above, for a fixed $r$, as $\mu$ increases, a single broadcast packet would be received correctly by more number of users inside the coverage area. Hence, the total number of encoded packets $t$ that needs to be sent in order to make sure that all the users successfully decode the message decreases. And when other parameters are fixed, as source packets $N$ increases, $\overline{C I}$ decreases.

Fig. 6 presents the effect of user density $\rho$ on $\overline{C l}$ and the approximation accuracy in Eq. (12). As one can see, on the one hand, increasing the value of $\rho$ increases $\overline{C I}$. On the other hand, increasing the value of $\rho$ decreases the gap between the simulation and approximation formulation in Eq. (12). In other words, larger $\rho$ results in better approximation accuracy and larger $\overline{C I}$. This is due to increasing $\rho$ is equivalent to increasing the number of users $K$, which consequently results in larger $\overline{C I}$. And increasing $\rho$ is more and more close to our assumption that users are uniformly distributed locating in rings centered at $\boldsymbol{S}$ with infinitesimal width and $K$ is large enough, which consequently results in better approximation.

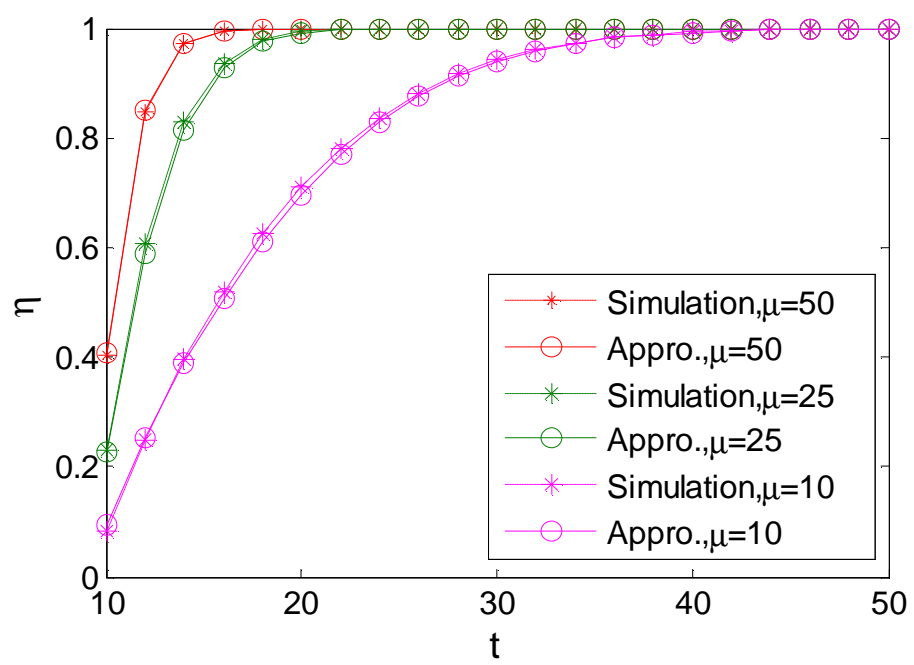

Fig. 4. Comparing the result of the approximation in Eq. (6) with computer simulation: the effect of fading parameter $\mu$ in our approximation model when $\rho=10, N=10$. 


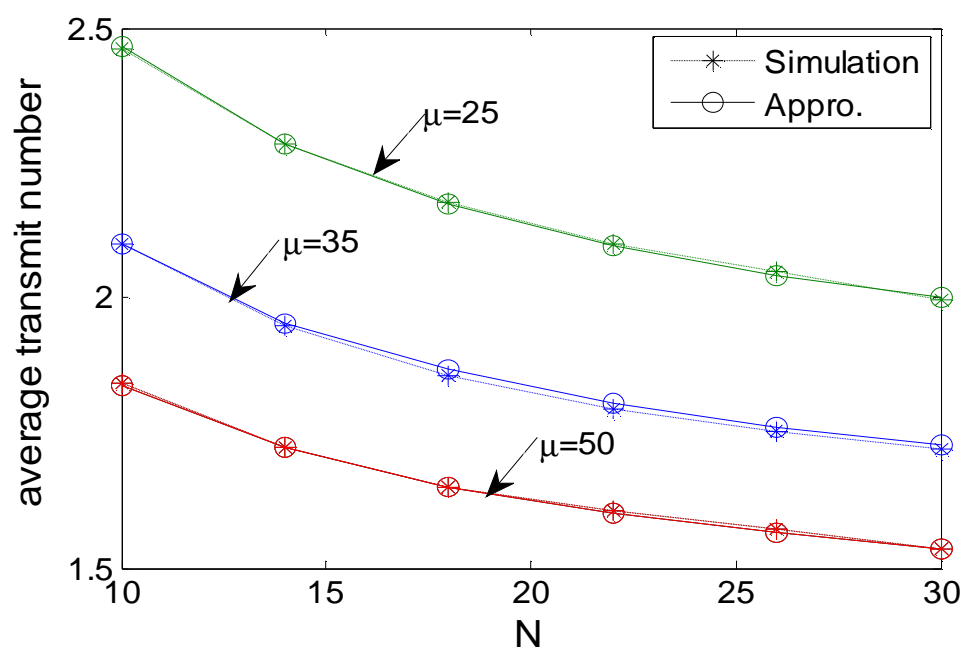

Fig. 5. Comparing the result of the approximation in Eq. (12) with computer simulation: The effect of fading parameter $\mu$ in our approximation model when $\rho=10$.

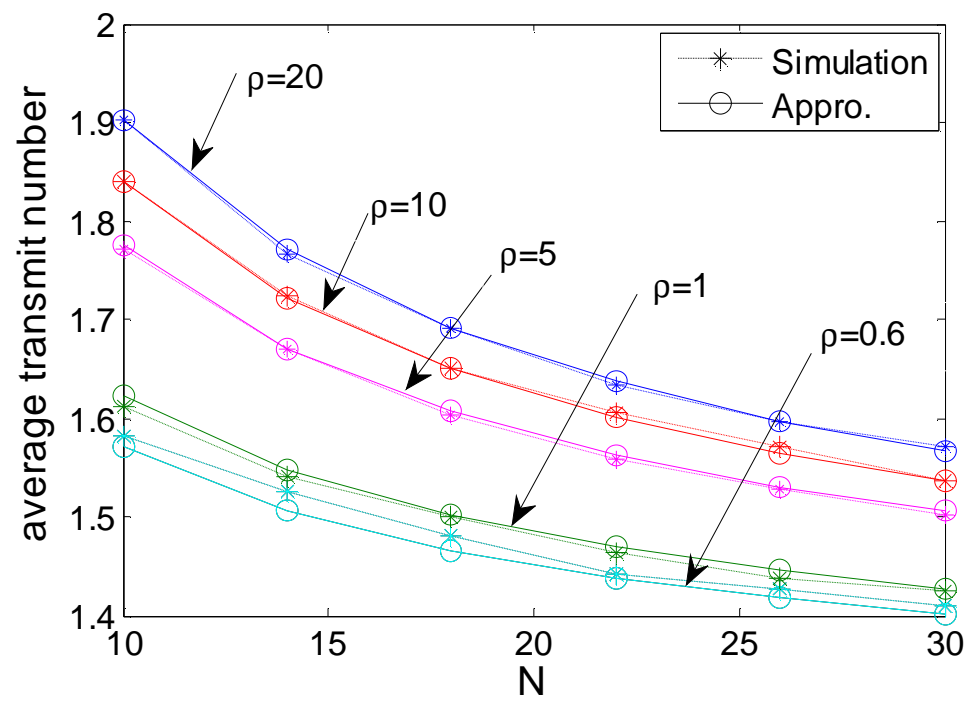

Fig. 6. Comparing the result of the approximation in Eq. (12) with computer simulation: The effect of user density $\rho$ in our approximation model when $\mu=50$.

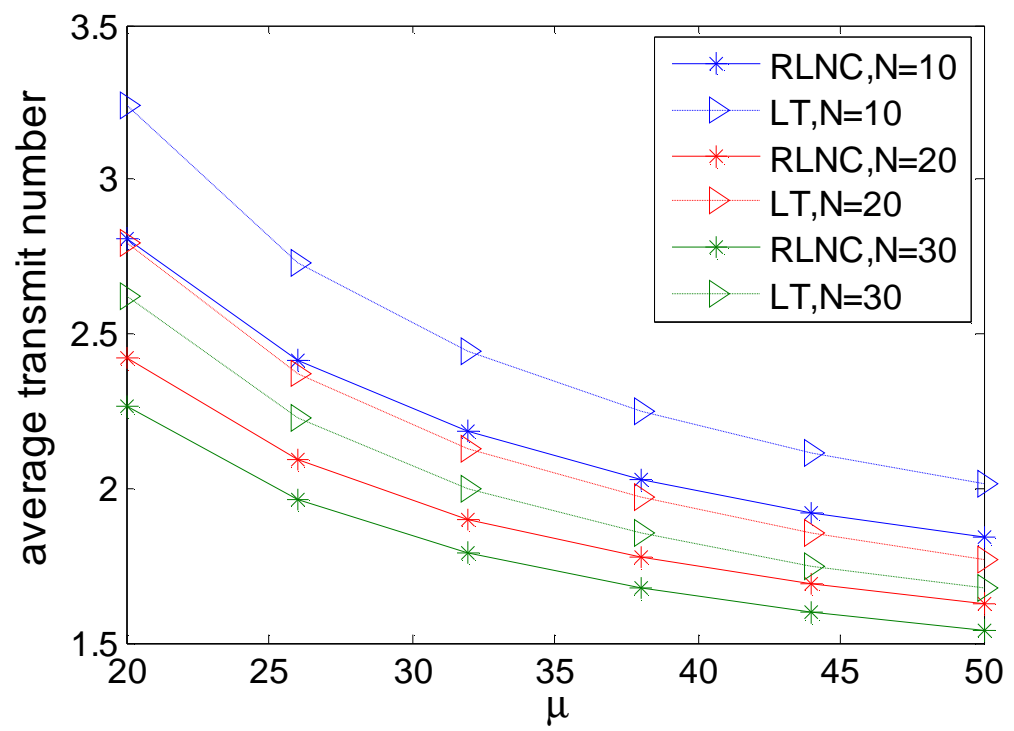

Fig. 7. Comparing the average number of required transmissions v.s. $\mu$ between RLNC and LT for $\rho=10$. 


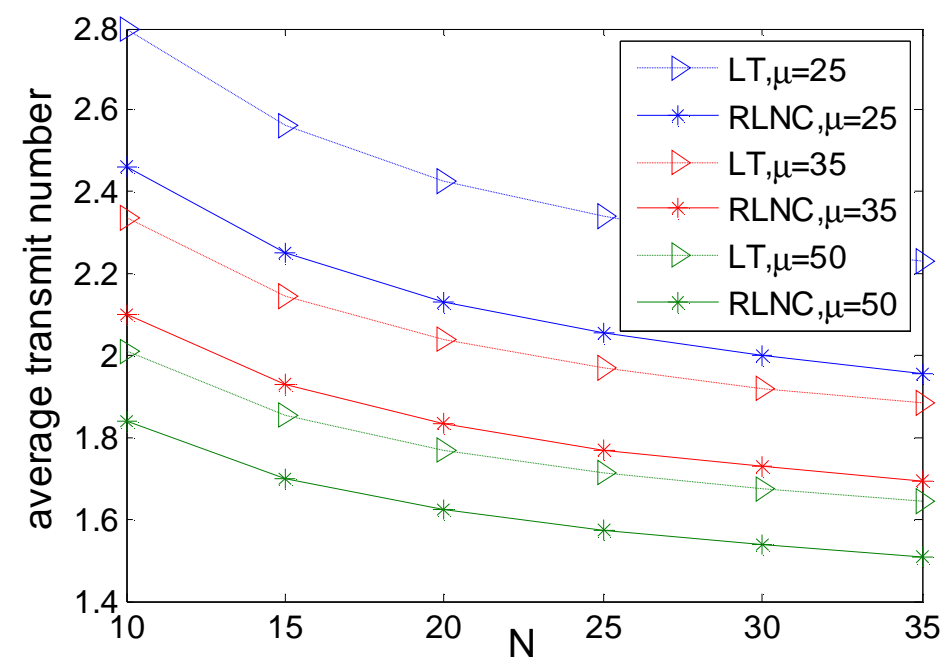

Fig. 8. Comparing the average number of required transmissions v.s. $N$ between RLNC and LT for $\rho=10$.

\section{B. RLNC Compare with LT Codes}

In this section, we compare the performance of RLNC based broadcast scheme with LT fountain codes. As can be seen from Fig. 7, RLNC based broadcast has smaller $\overline{C I}$ than LT encoding for a given block fading parameter $\mu$. Fig. 8 shows that RLNC based broadcast always outperforms LT encoding for a given source packets $N$. The reason is that RLNC combines the packets in Galois Field $F_{q}(q$ is large enough), while in traditional LT codes, source packets are combined over a binary field (or Galois field $F_{2}$ ). That means there are more degrees of freedom in constructing the coding matrix $\boldsymbol{C}$ in RLNC scheme. As a result, it has a greater opportunity to have a full rank coding matrix when RLNC is employed as erasure channel coding, which leads to a higher probability of successful decoding and smaller $\overline{C l}$.

\section{Conclusions}

In this paper, we have analyzed the performances of the RLNC based wireless broadcasting system, where a source wants to broadcast $N$ packets to a large number of users with uniform distribution. Furthermore, by resorting to stochastic geometry analysis, the approximate average number of transmissions and the average percent of successful decoding users in the RLNC based wireless broadcasting system with uniformly distributed users have been derived. Simulation results have shown the accuracy of our theoretical analysis and that a user can recover all the original packets with high probability by receiving $N$ out of all the transmitted RLNC combined packets. The new analysis results can guide the system designs in practice. Meanwhile, we have demonstrated the viability of RLNC as an efficient broadcast mechanism .

\section{Acknowledgment}

This work is supported by Major Research Plan of NationalNatural Science Foundation of China (No. 91438115), National Natural Science Foundation of China (No. 61371123, No.61301165), Special Financial Grant of The China Postdoctoral Science Foundation (2015T81079), China Postdoctoral Science Foundation (2014M552612) and Preresearch ProjectFoundation(9140C020306130C02007).

\section{References}

[1] Y. Xu, X. Xia, K. Xu, and D. Zhang, On the hybrid relaying protocol for time division broadcasting. Trans. Emerging Telecommun. Tech., 26(2015) 893-904. 
[2] A. Shokrollahi, "Raptor codes,” IEEE Trans. Inf. Theory, 52(2006) 2551-2567.

[3] M. Luby, "LT codes," in Proc. 43rd Ann. IEEE Symp. Found. Comput.Sci., (2002) 271-280.

[4] S.Y. R. Li, R. W. Yeung, and N. Cai, "Linear network coding," IEEE Trans. on Inf. Theory, 49(2003) 371-381.

[5] R. Koetter and M. Medard, "An algebraic approach to network coding," IEEE/ACM Trans. Networking, 11(2003) 782-795.

[6] S.M.Choi and J.S.Park, "Massive Parallelization technique for Random Linear Network Coding," in IEEE Big Data and Smart Computing(BIGCOMP), (2014) 296-299.

[7] H.Xi, X.Wang, Y. Zhao, and H.Zhang, "A Reliable Broadcast Transmission Approach Based on Random Linear Network Coding" IEEE Vehicular Technology Conference (VTC Spring) 75th, (2012).

[8] B.T.Swapna, Atilla Eryilmaz, and Ness B.Shroff, "Throughput-Delay Analysis of Random Linear Network Coding for Wireless Broadcasting” IEEE Trans. Inf. Theory, 59(2013).

[9] M.H.Firooz, Zixia Hu, and Sumit Roy, "Random Linear Network Coding for Reliable Broadcasting," in IEEE Globecom Communications QoS, Reliability and Modelling Symposium on (2014) 1237-1241.

[10] A.Goldsmith, Wireless communications. Cambridge university press, (2005).

[11] D.MacKay, "Fountain codes," in IEEE Proc. Communications, 152(2005) 1062-1068.

[12] T.Ho, "Networking from a network coding perspective," Ph.D. dissertation, Massachusetts Inst. Technol., Cambridge, MA, USA, (2004). 